\title{
OPTIMISING ACCELERATION AND BRAKING PERFORMANCE PROFILES OF RAILWAY VEHICLES FOR THE ROBUST OPERATION OF ULTRA-HIGH FREQUENCY TIMETABLES
}

\author{
RYO TAKAGI \& MASAO WATANABE \\ Department of Electrical and Electronic Engineering, Kogakuin University, Japan
}

\begin{abstract}
Ultra-high frequency train timetables, which will double the capacity of urban railway lines, are expected to eliminate the chronic congestion and minimise passengers' disbenefit. However, it is well known that the higher the train frequency, the lower the so-called "robustness" or "resilience" of the timetable. Also, it is generally accepted that the fundamental, and in many cases the only, method to improve robustness and/or resilience is to add larger buffer times to it. This will cause disbenefits for the operators and the passengers alike because this will make trains slower. Instead, we examined the method to replace the vehicles with the ones with considerably higher acceleration and braking performance. According to our findings, this method is effective in allowing larger buffer times without slowing down trains for most cases but, unfortunately, not always. Also, problems in the trackside power supply system, such as overloaded substations, excessive voltage drop or too many losses, are almost inevitable because the power consumed or regenerated by trains become too high. Through these investigations, we concluded that the optimisation of the acceleration and braking performance of trains was necessary. However, few literatures were found on this problem. Many previous works on the optimisation of running profiles of trains could be found. In these optimisation attempts, however, the performance of trains was treated as the "given" condition for them. In this paper, we discuss the impact of the acceleration/braking performance of the railway vehicles on the whole system performance, based on the results of both the literature survey and some calculations. We then discuss how train acceleration/braking performance optimisation can be performed.
\end{abstract}

Keywords: railway timetabling, robustness, resilience, buffer time, accelerating and braking performance of railway vehicles.

\section{INTRODUCTION}

Ultra-high frequency train timetables, such that will double the capacity of urban railway lines, are expected to eliminate the chronic congestion of urban railways and minimise passengers' disbenefit [1]. However, it is well known that the higher the frequency, the lower the so-called "robustness" or "resilience" of the timetable as defined by Goverde and Hansen [2]. This is mainly due to the fact that a small delay of a train will affect movements of larger number of trains as train frequency goes up. It is also generally accepted that the fundamental, and in many cases the only, method to improve robustness and/or resilience of a train timetable is to add larger buffer times to it. Increasing buffer times generally results in trains running slower, which of course causes disbenefit both for the operators and for the passengers.

Buffer times can be defined as the difference between the scheduled and the fastest possible run times of trains between the same pair of stations. Increasing buffer times can be realised by either extending the scheduled run times or cutting the fastest possible run times. Although extending the scheduled run times is clearly easier, it will result in slower timetables. If we can consider replacing the vehicles with those having considerably higher accelerating and braking performance, it may result in shorter fastest run times and hence larger buffer times, even when the scheduled run times are unchanged. 
However, this replacement of vehicles may not yield good enough reduction in fastest run times. Also, this method may result in high power demands by the accelerating trains, causing overload at the traction substations. Therefore, assessment of the run time reductions and the impact on traction system loadings, energy consumption, etc., is required when considering such a replacement.

In this paper, we first explain we explain what the ultra-high frequency train operation is and how this can be achieved. Then we discuss the impact of ultra-high frequency operation on the timetable "resilience", and the need for increasing buffer times. Finally, we present a set of preliminary calculations to verify: (a) how buffer times can be increased by higher accelerating and braking performance of the railway vehicles, and (b) the impact on the traction power supply systems and energy consumption.

\section{WHAT IS ULTRA-HIGH FREQUENCY TRAIN OPERATION AND HOW IT CAN BE REALISED}

For urban railways using long trainsets $(\sim 200 \mathrm{~m})$, the practical maximum train frequency is around 30 tphpd (trains per hour per direction). On many commuting railway lines in large cities like Tokyo, unacceptable level of congestion is observed even with this level of frequent train operation, and the increase in transport capacity is desperately needed.

In this paper, the word "ultra-high frequency train operation" refers to the operation that will realise the transport capacity that is nearly twice as much as the currently realised level. This will mean the frequency level close to 60 tphpd when trains of the same length on the same railway line are assumed, or even higher frequency if the train lengths are to be shortened.

Such frequency will be realised by the introduction of very complicated scheduling schemes. For example, assume there is a railway line where the simple "parallel and local only" scheduling scheme, i.e. the scheduling scheme in which all trains are local trains that will call at every station en route, is applied when planning its train timetable. If there is, on this line, an intermediate station with only one platform per direction where all trains should dwell for more than 60 seconds due to a very high demand, it would be very difficult to raise the train frequency higher than 30 tphpd.

In fact, such stations can be found on many existing commuting lines in Tokyo. Those stations form the bottleneck on these lines, where the minimum headway between trains is limited to around 130 seconds, which yields 27 tphpd.

If we can assume that half of the trains can pass this station, then it would be possible to think of doubling the frequency. Fig. 1 [3] shows that the headway between two trains stopping at a station, trains \#1 and \#3, can be maintained at $130 \mathrm{~s}$ while another train, train $\# 2$, can be inserted in between these two trains so that the capacity is doubled to 54 tphpd. The calculation shown in Fig. 1 is based on the following set of assumptions: (1) pure moving block signalling is introduced, (2) vehicles are designed capable of constant acceleration and deceleration of $3.6 \mathrm{~km} /(\mathrm{h} \cdot \mathrm{s})$ or $1 \mathrm{~m} / \mathrm{s}^{2}$ regardless of their speed, and (3) the passing train is allowed to make an out-of-station halt before entering the platform.

The resulting timetable for the entire line would be much more complicated than the current one based on the "parallel and local only" scheduling scheme, especially if the possibility is to be maintained for passengers to travel between any pair of stations without having to use trains running in the opposite direction. Therefore, the implementation of a sophisticated system of guidance for passengers, such as the "IPASS" concept as proposed by Takagi [4], would be crucial in the realisation of this kind of timetables. Even with these issues, the doubled transport capacity will be a clear benefit to all users of this railway. 
It should also be noted that, even in the central area of large cities such as Tokyo, stations with such high demands are very limited. Investment in infrastructure, such as increasing platforms at these stations, in combination with the introduction of the complicated scheduling scheme and the passenger assistance measures such as IPASS, is expected to yield even better timetables.

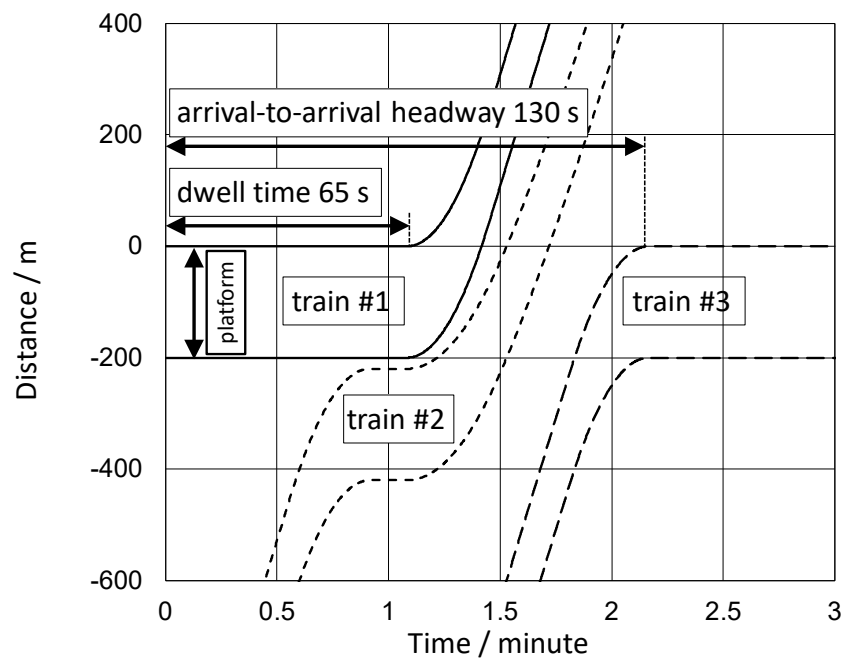

Figure 1: Train movement of an example ultra-high frequency operation at a large station with insufficient platforms.

\section{THE TIMETABLE RESILIENCE OF ULTRA-HIGH FREQUENCY TRAIN OPERATION AND THE NEED TO INCREASE BUFFER TIMES}

An ultra-high frequency train timetable is, however, believed by the train operators to be difficult to operate, especially because it is susceptible to disruptions. In such a timetable, the initial small disruptions that frequently occur, such as those caused by passengers or their belongings being caught in opening or closing doors, will easily grow into large disruptions as they create secondary delays to more trains than in the conventional timetables. These initial small disruptions are very difficult to prevent, and will be more frequent as congestion gets worse.

Therefore, for an ultra-high frequency timetable to be operable successfully, it must have enough buffer times. The buffer time for a scheduled run of a train from any one station to another is, as has been explained in Section 1, defined as the difference between the scheduled run time and the fastest possible run time of that train. The fastest possible run time is dependent on the track profile and the accelerating and braking performance of the vehicles to be used with the train, and is not easily shortened. It is much easier to extend the scheduled run times to realise larger buffer times; however, this will lead to slower timetables.

According to the existing timetables on the commuting lines in Tokyo with very heavy peak-hour traffic, trains are running significantly slower in those peak hours during which the train frequency is higher than in other hours of the day. Of course, the "high" frequency during peak hours on these lines is not "ultra-high" as we expect in this paper. We can 
therefore safely deduce from this observation that adding buffer times even further is crucially needed when realising the ultra-high frequency operation which has enough "robustness" or "resilience".

At the same time, it is undesirable to slow trains even further to increase buffer times from the point of view of the passengers' and operators' disbenefits. Although not an easy option, replacing the railway vehicles currently in use with higher accelerating and braking performance to shorten the fastest possible run times for realising the buffer time increase without slowing trains should be considered.

\section{DISCUSSION ON HOW TO ENHANCE ACCELERATING AND BRAKING PERFORMANCE OF RAILWAY VEHICLES}

Generally, the accelerating performance of the railway vehicles can be considered as the combination of three different modes, namely: (a) "constant-torque" at low speed region, where the torque of the traction motors, and hence the tractive effort of the vehicles, is constant regardless of their speed; (b) "constant-power" at middle speed region, where the output power of the vehicles at wheel rim is constant regardless of their speed; and (c) "free-run" at high speed region, where the output power of the vehicles is in inverse proportion to their speed. Out of these three, the speed region for (c) is sometimes considered as above the practical maximum speed of the vehicles. The tractive effort at region (a) is constrained by the limit of adhesion, while the power at region (b) is limited by the rated output of the traction equipment onboard the trains.

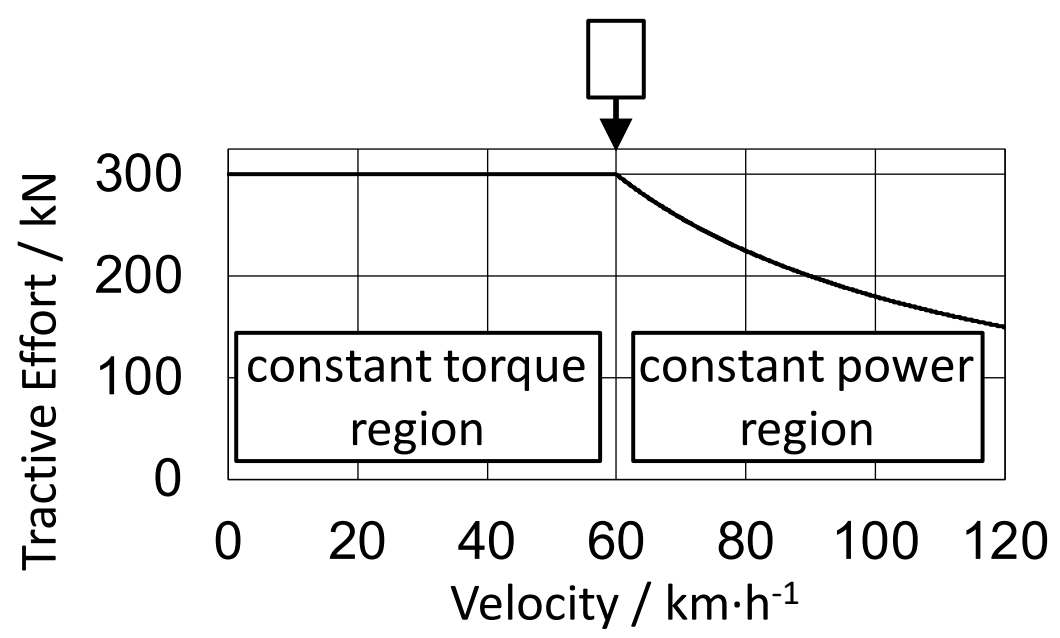

Figure 2: Train movement of an example ultra-high frequency operation at a large station with insufficient platforms.

Fig. 2 shows an example tractive effort-speed profile of a vehicle with only two regions, (a) and (b), under the assumption that the tractive effort at (a) and the output power at (b) are $300 \mathrm{kN}$ and $5000 \mathrm{~kW}$, respectively. The parameter $v_{1}$ in the figure shows the boundary speed between the two regions, which becomes $60 \mathrm{~km} / \mathrm{h}$ under the above assumptions.

Let us discuss how the enhancement of accelerating performance of the vehicles can be done using the performance profile of Fig. 2. The possible methods are: 
1. Increasing the tractive effort at region a;

2. Increasing the output power at region $b$; and

3. Doing both (1) and (2).

Method (1) requires better utilisation of adhesion. One possible way to achieve this is by increasing the ratio of the number of driving axles to the number of all axles in a train set. Because the output power at region $b$, and therefore the vehicle performance at the higher speed region, remain unchanged, $v_{1}$ in Fig. 2 becomes lower as the tractive effort increases. On the other hand, method (2) requires increasing the power output of the traction equipment. Because the tractive effort at region a, and therefore the vehicle performance at the lower speed region, remain unchanged, v1 in Fig. 2 becomes higher as the output power increases. Method (3) is the combination of two methods, and therefore enhancement of performance at all speed regions is realised.

Here, it must be noted that, to achieve meaningful increase in buffer times, reduction of the fastest run times between the adjacent stations by a couple of seconds at least should be realised. Also note that the discussion in this paper is mainly for urban railways, where the distance between any adjacent stations is short (e.g. $1 \mathrm{~km})$. Therefore, the necessary enhancement of the performance will be a significant one. If the output power of the vehicles are to be significantly increased as methods (2) and/or (3) are to be implemented, very high overload may occur at the traction substations as the result. It is unlikely that method (1) causes the similar kind of problem, but the fact that the performance at higher speed region will not be enhanced may mean insufficient reduction of the fastest run times.

\section{PRELIMINARY EVALUATION OF THE RUN TIME REDUCTION}

We have performed a number of preliminary calculations to see how much the fastest inter-station run times of a train can be reduced by method (1) in the previous section.

Assumed is a 200-m-long, 313-t train set with the interior design which is typically seen in Japan, fully loaded with passengers, and with the traction equipment having the output power at wheel rim of $5500 \mathrm{~kW}$. Assumed acceleration at region a are: $2.5 \mathrm{~km} /(\mathrm{h} \cdot \mathrm{s})(=0.69$ $\left.\mathrm{m} / \mathrm{s}^{2}\right), 3.0 \mathrm{~km} /(\mathrm{h} \cdot \mathrm{s})\left(=0.83 \mathrm{~m} / \mathrm{s}^{2}\right), 3.5 \mathrm{~km} /(\mathrm{h} \cdot \mathrm{s})\left(=0.97 \mathrm{~m} / \mathrm{s}^{2}\right), 4.0 \mathrm{~km} /(\mathrm{h} \cdot \mathrm{s})\left(=1.11 \mathrm{~m} / \mathrm{s}^{2}\right), 4.5$ $\mathrm{km} /(\mathrm{h} \cdot \mathrm{s})\left(=1.25 \mathrm{~m} / \mathrm{s}^{2}\right), 5.0 \mathrm{~km} /(\mathrm{h} \cdot \mathrm{s})\left(=1.39 \mathrm{~m} / \mathrm{s}^{2}\right)$ and $5.5 \mathrm{~km} /(\mathrm{h} \cdot \mathrm{s})\left(=1.53 \mathrm{~m} / \mathrm{s}^{2}\right)$. For deceleration, the same value as the acceleration in region a is assumed for the entire speed region. The track profile is assumed as straight and level all the way. The train is to start from halt at the origin station, accelerate, decelerate and finally come to a complete halt at the destination station. In the calculation, infinite jerk is allowed. When the train speed reaches $100 \mathrm{~km} / \mathrm{h}$ during acceleration, the train is assumed to stop the acceleration and keep this speed of $100 \mathrm{~km} / \mathrm{h}$ until it starts braking in order to stop at the appropriate position in the destination station.

Table 1 shows some of the calculation results. The difference between the run time for the acceleration of $2.5 \mathrm{~km} /(\mathrm{h} \cdot \mathrm{s})$ and the run time for one of the other acceleration values will be the reduction in the run times when raising the acceleration at region a from 2.5 to the selected value. As an example, the difference between the $2.5 \mathrm{~km} /(\mathrm{h} \cdot \mathrm{s})$ case and the $5.5 \mathrm{~km} /(\mathrm{h} \cdot \mathrm{s})$ case for each assumed distance has been calculated and added at the bottom row of Table 1 .

It is generally expected that, as the inter-station distance becomes longer, this difference becomes larger. However, looking at the calculation results, the differences seem more or less independent of the inter-station distance; This is due to: (1) the acceleration performance at higher speed is unchanged, and (2) the maximum speed reaches the assumed maximum of $100 \mathrm{~km} / \mathrm{h}$ in many calculated cases. Especially, because of (2), this difference when even 
longer inter-station distance is assumed is almost identical to the case with the assumed inter-station distance of $1400 \mathrm{~m}$.

Table 1: Calculated fastest inter-station run times over the assumed distances.

\begin{tabular}{|c|c|c|c|c|c|}
\hline \multirow{2}{*}{ Acceleration and deceleration } & \multicolumn{5}{|c|}{ Inter-station distance } \\
\cline { 2 - 6 } & $600 \mathrm{~m}$ & $800 \mathrm{~m}$ & $1000 \mathrm{~m}$ & $1200 \mathrm{~m}$ & $1400 \mathrm{~m}$ \\
\hline $2.5 \mathrm{~km} /(\mathrm{h} \cdot \mathrm{s})$ & $59.02 \mathrm{~s}$ & $68.47 \mathrm{~s}$ & $76.97 \mathrm{~s}$ & $84.79 \mathrm{~s}$ & $92.10 \mathrm{~s}$ \\
\hline $3.0 \mathrm{~km} /(\mathrm{h} \cdot \mathrm{s})$ & $54.46 \mathrm{~s}$ & $63.49 \mathrm{~s}$ & $71.64 \mathrm{~s}$ & $79.16 \mathrm{~s}$ & $86.37 \mathrm{~s}$ \\
\hline $3.5 \mathrm{~km} /(\mathrm{h} \cdot \mathrm{s})$ & $51.24 \mathrm{~s}$ & $59.99 \mathrm{~s}$ & $67.91 \mathrm{~s}$ & $75.25 \mathrm{~s}$ & $82.45 \mathrm{~s}$ \\
\hline $4.0 \mathrm{~km} /(\mathrm{h} \cdot \mathrm{s})$ & $48.88 \mathrm{~s}$ & $57.42 \mathrm{~s}$ & $65.18 \mathrm{~s}$ & $72.43 \mathrm{~s}$ & $79.63 \mathrm{~s}$ \\
\hline $4.5 \mathrm{~km} /(\mathrm{h} \cdot \mathrm{s})$ & $47.07 \mathrm{~s}$ & $55.47 \mathrm{~s}$ & $63.10 \mathrm{~s}$ & $70.31 \mathrm{~s}$ & $77.51 \mathrm{~s}$ \\
\hline $5.0 \mathrm{~km} /(\mathrm{h} \cdot \mathrm{s})$ & $45.66 \mathrm{~s}$ & $53.93 \mathrm{~s}$ & $61.47 \mathrm{~s}$ & $68.67 \mathrm{~s}$ & $75.86 \mathrm{~s}$ \\
\hline $5.5 \mathrm{~km} /(\mathrm{h} \cdot \mathrm{s})$ & $44.51 \mathrm{~s}$ & $52.70 \mathrm{~s}$ & $60.15 \mathrm{~s}$ & $67.35 \mathrm{~s}$ & $74.55 \mathrm{~s}$ \\
\hline $\begin{array}{l}\text { Difference }(5.5 \mathrm{~km} /(\mathrm{h} \cdot \mathrm{s}) \\
\text { case }-2.5 \mathrm{~km} /(\mathrm{h} \cdot \mathrm{s}) \mathrm{case})\end{array}$ & $14.51 \mathrm{~s}$ & $15.77 \mathrm{~s}$ & $16.82 \mathrm{~s}$ & $17.44 \mathrm{~s}$ & $17.55 \mathrm{~s}$ \\
\hline
\end{tabular}

\section{DISCUSSIONS}

The acceleration of $2.5 \mathrm{~km} /(\mathrm{h} \cdot \mathrm{s})$ or $3.0 \mathrm{~km} /(\mathrm{h} \cdot \mathrm{s})$ are not particularly high for the urban railway vehicles, but are the commonly seen values in many commuting lines in Tokyo. $5.5 \mathrm{~km} /(\mathrm{h} \cdot \mathrm{s})$ is the value typical for tram and light rail vehicles rather than the vehicles for heavy metro lines, and therefore there is no reason why this high acceleration cannot be adopted for heavy metro trains. Therefore, although at the preliminary stage, it seems we can conclude that raising the acceleration at region a, i.e. at low speed region, without increasing the maximum output power, is effective in reducing run times. The run time reduction, of course, can be used to increase buffer times by keeping the scheduled run times to the level achievable by the current vehicles with lower performance.

However, the following should be considered.

\subsection{Proper consideration of track profiles are necessary}

In the calculations in Section 5, track profiles are assumed as straight and level, and no speed restriction has been taken into account. This is, of course, very unrealistic; we have to take up a more realistic model to evaluate the possibility.

\subsection{Constant deceleration is assumed for the entire speed region}

Also, constant deceleration has been assumed for the entire speed region. This means that, for the cases with higher assumed acceleration, such as $5 \mathrm{~km} /(\mathrm{h} \cdot \mathrm{s})$, the maximum braking power will also become very high (for the case of $5 \mathrm{~km} /(\mathrm{h} \cdot \mathrm{s}$ ), it will become twice as much as the case for $2.5 \mathrm{~km} /(\mathrm{h} \cdot \mathrm{s})$ ). Designing a traction system that can absorb this level of high regenerative power will be a very difficult work. 


\subsection{The nature of ultra-high frequency timetables: Trains are to call at fewer stations}

In Section 2, we discussed that the ultra-high frequency timetables are complicated timetables in terms of the patterns of station calls made by trains. However, from another perspective, they can be called as the timetables in which trains make much fewer calls at stations. This means that, for at least some of the trains in these timetables, the "start-to-stop" run times between stations that are close by may not be the issue. We may have to consider cases in which trains which pass many stations are requested to control their inter-station run times, for which acceleration performance at the middle to high speed region may become more important.

As has been discussed in Section 4, this requires the adoption of methods (2) or (3). However, the required output power of the train becomes large, which have a very significant impact on the trackside traction power supply system. Already, we have performed some simulations on the idea to increase power of trains so that the robustness or resilience is improved [5]. However, it was quickly revealed that the high performance led to the overload of substations. A number of countermeasures, including the method to allow the use of high power only when the train is delayed and needs it for recovery, have been tried but without satisfactory results in suppressing substation loads to the safe level. We believe that the application of techniques proposed by Takagi and Sone [6] will help when it is implemented together with the introduction of very-high-power vehicles.

\subsection{Optimisation of the vehicle performance should be attempted}

From the discussions in 6.3, obviously there is a need for optimisation of the acceleration and braking performance of the railway vehicles.

There have been a number of attempts to optimise the operational pattern of a train for an inter-station run. One of the most impressive examples in which this kind of optimisation has been performed would be the one presented by Ko and Miyatake [7]. However, in this kind of research, the train performance is the assumption and not the parameters to be optimised.

We could find very few works have been found in which optimisation of the railway vehicle performance is performed in any way. In one example [8], simulations are repeated for railway vehicles with different specifications for the traction equipment on board. Among the conclusions therein is the finding that, from the point of view of energy saving, different optimal designs of the traction equipment exist for different operating conditions, especially the inter-station start-to-stop run times.

For the purpose of the ultra-high frequency train operation, we should perform optimisations taking into account not only the energy consumption but also the load limitations of the trackside power supply network.

\subsection{Proper consideration of timetable resilience is required}

Throughout this paper, we have not distinguished timetable "robustness" and timetable "resilience", although Goverde and Hansen [2] made a clear distinction between these two.

According to Goverde and Hansen [2], timetable robustness is the ability of a timetable to withstand design errors, parameter variations, and changing operational conditions, while timetable resilience is the flexibility of a timetable to prevent or reduce secondary delays using dispatching (re-timing, re-ordering, re-routing). Generally, adding buffer times are directly connected to timetable robustness. We understand that the improvement in timetable robustness will lead to the improvement in timetable resilience. 
However, it must also be noted that the complicated nature of the ultra-high frequency timetable as outlined in Section 2 may make train control (dispatching) very difficult. Although we can generally state that more buffer times will open up wider possibility for good train control, the relationship with the buffer times and the timetable resilience should be seriously sought, together with the effective and easy-to-perform method of train control operations.

\section{CONCLUSIONS}

We have investigated the possibility of increasing buffer times of the ultra-high frequency train timetables by means of significantly enhancing the accelerating and braking performance of railway vehicles to be used with them. The preliminary calculation results give hope that significantly higher performance leads to effective reduction in the fastest run times, leading to more buffer times and hence more resilient operation.

Ultra-high frequency train operation, once realised, will give clear benefits to passengers, rail operators and the wider society. Although we are still at the preliminary stage and there are still so many challenges to be tackled as listed in Section 6, through the future works we believe we can move this idea closer to reality from the train control points of view.

\section{REFERENCES}

[1] Shimizu, T., Watanabe, M. \& Takagi, R., Scheduling techniques to achieve ultra-high frequency operation over existing infrastructure for realization of ultra-convenient rail transport. Proceedings of International Symposium on Speed-up and Service Technology for Railway and Maglev Systems (STECH 2015), Chiba, Japan, 3D24, 2015.

[2] Goverde, R.M.P. \& Hansen, I.A., Performance indicators for railway timetables. IEEE International Conference on Intelligent Rail Transportation (IEEE ICIRT 2013), Beijing, China, 2013.

[3] Takagi, R., You Can Make a Railway Timetable, Ohmsha Ltd.: Tokyo, pp. 169-175, 2019. (In Japanese.)

[4] Takagi, R., Reservation-compulsory commuting railways: Innovation that will be made possible by UCRT/IPASS. WIT Transactions on the Built Environment, vol. 181, WIT Press: Southampton and Boston, pp. 225-233, 2018.

[5] Watanabe, M., Shimizu, T. \& Takagi, R., Measures against disruptions on ultra-high frequency operated railways: operation of high performance vehicles and substation peak load suppression. IEEJ Joint Technical Workshop on Physical Sensors/ Transportation \& Electric Railways, TER-16-018/PHS-16-012, Nagoya, Japan, 2016. (In Japanese.)

[6] Takagi, R. \& Sone, S., More intelligent DC railway electrical power systems with traction power control. Electrical Engineering in Japan, 114(7), pp. 91-101, 1994.

[7] Ko, H. \& Miyatake, M., A numerical algorithm for run-curve optimization of trains considering a DC feeding circuit. WIT Transactions on the Built Environment, vol. 88, WIT Press: Southampton and Boston, pp. 787-796, 2006.

[8] The energy economy of train operations and the specifications of traction equipment for the railway vehicles. IEEJ Technical Report, 112(2), 1981. (In Japanese.) 